\title{
Exponential Stability of BAM Fuzzy Cellular Neural Networks with Time-Varying Delays in Leakage Terms and Impulses
}

\author{
Yongkun Li and Youqin Li \\ Department of Mathematics, Yunnan University, Kunming, Yunnan 650000, China \\ Correspondence should be addressed to Yongkun Li; yklie@ynu.edu.cn
}

Received 23 September 2013; Revised 25 January 2014; Accepted 27 January 2014; Published 17 March 2014

Academic Editor: Jaeyoung Chung

Copyright ( 2014 Y. Li and Y. Li. This is an open access article distributed under the Creative Commons Attribution License, which permits unrestricted use, distribution, and reproduction in any medium, provided the original work is properly cited.

BAM fuzzy cellular neural networks with time-varying delays in leakage terms and impulses are considered. Some sufficient conditions for the exponential stability of the networks are established by using differential inequality techniques. The results of this paper are completely new and complementary to the previously known results. Finally, an example is given to demonstrate the effectiveness and conservativeness of our theoretical results.

\section{Introduction}

The bidirectional associative memory (BAM) neural networks were first introduced by Kosko [1-3]. It is a special class of recurrent neural networks that can store bipolar rector pairs. The BAM neural networks are composed of neurons arranged in two layers, the X-layer and Y-layer. Recently, many researchers have studied the dynamics of BAM neural networks with or without delays [4-15]. However, in mathematical modeling of real world problems, uncertainty or vagueness is unavoidable. In order to take vagueness into consideration, fuzzy theory is considered as a suitable method. In $[16,17]$, the authors first combined those operations with cellular neural networks (FCNNs). Some results have been reported on stability and periodicity of FCNNs. More recently, state estimation problem for the fuzzy BAM neural networks has been obtained in the paper $[18,19]$ and passivity criteria for the fuzzy BAM neural networks have been studied in the papers [20,21].

Very recently, a leakage delay, which is the time delay in leakage term of the systems and a considerable factor affecting dynamics for the worse in the systems, is being put to use in the problem of stability for neural networks $[22,23]$. However, so far, very little attention has been paid to neural networks with time delay in the leakage (or "forgetting") term [24-30]. Such time delays in leakage terms are difficult to handle but have great impact on the dynamical behavior of neural networks.
In [31], the authors studied the following BAM fuzzy cellular network with time delay in leakage terms and discrete and unbounded distributed delays:

$$
\begin{aligned}
x_{i}^{\prime}(t)= & -d_{i} x_{i}\left(t-\sigma_{1}\right)+\sum_{j=1}^{m} a_{i j} \widetilde{f}_{j}\left(y_{j}(t)\right) \\
& +\sum_{j=1}^{m} b_{i j} \widetilde{f}_{j}\left(y_{j}(t-\tau(t))\right)+\sum_{j=1}^{m} c_{i j} \omega_{j} \\
& +\bigwedge_{j=1}^{m} \alpha_{i j} \int_{-\infty}^{t} k_{j}(t-s) \tilde{f}_{j}\left(y_{j}(s)\right) d s \\
& +\bigvee_{j=1}^{m} \beta_{i j} \int_{-\infty}^{t} k_{j}(t-s) \widetilde{f}_{j}\left(y_{j}(s)\right) d s \\
& +\bigwedge_{j=1}^{m} T_{i j} \omega_{j}+\bigvee_{j=1}^{m} H_{i j} \omega_{j}+I_{i}, \\
& t \geq 0, \quad i=1,2, \ldots, n, \\
y_{j}^{\prime}(t)= & -\widetilde{d}_{j} y_{j}\left(t-\sigma_{2}\right)+\sum_{i=1}^{n} \widetilde{a}_{j i} \widetilde{g}_{i}\left(x_{i}(t)\right) \\
& +\sum_{i=1}^{n} \widetilde{b}_{j i} \widetilde{g}_{i}\left(x_{i}(t-\rho(t))\right)+\sum_{i=1}^{n} \widetilde{c}_{j i} \widetilde{\omega}_{i}
\end{aligned}
$$




$$
\begin{aligned}
& +\bigwedge_{i=1}^{n} \widetilde{\alpha}_{j i} \int_{-\infty}^{t} k_{i}(t-s) \widetilde{g}_{i}\left(x_{i}(s)\right) d s \\
& +\bigvee_{i=n}^{m} \widetilde{\beta}_{j i} \int_{-\infty}^{t} k_{i}(t-s) \widetilde{g}_{i}\left(x_{i}(s)\right) d s \\
& +\bigwedge_{i=1}^{n} \widetilde{T}_{j i} \widetilde{\omega}_{i}+\bigvee_{i=1}^{n} \widetilde{H}_{j i} \widetilde{\omega}_{i}+J_{j}, \\
& t \geq 0, \quad j=1,2, \ldots, m .
\end{aligned}
$$

However, time-varying delays in the leakage terms inevitably occur in electronic neural networks owing to the unavoidable finite switching speed of amplifiers. It is desirable to study the fuzzy BAM neural networks with time-varying delays in leakage terms. In [32], by using a fixed point theorem and differential inequality techniques, the authors studied the existence and exponential stability of equilibrium point for the following BAM neural network with time-varying delays in leakage terms on time scales:

$$
\begin{aligned}
x_{i}^{\Delta}(t)= & -a_{i} x_{i}\left(t-\delta_{i}(t)\right)+\sum_{j=1}^{m} c_{j i} f_{j}\left(y_{j}\left(t-\tau_{j i}(t)\right)\right) \\
& +\bigwedge_{j=1}^{m} \alpha_{j i} f_{j}\left(y_{j}\left(t-\tau_{j i}(t)\right)\right) \\
& +\bigwedge_{j=1}^{m} T_{j i} \mu_{j}+\bigvee_{j=1}^{m} \beta_{j i} f_{j}\left(y_{j}\left(t-\tau_{j i}(t)\right)\right) \\
& +\bigvee_{j=1}^{m} H_{j i} \mu_{j}+I_{i}, \quad t \in \mathbb{T}, i=1,2, \ldots, n, \\
y_{j}^{\Delta}(t)= & -b_{j} y_{j}\left(t-\eta_{j}(t)\right)+\sum_{i=1}^{n} d_{i j} g_{i}\left(x_{i}\left(t-\sigma_{i j}(t)\right)\right) \\
& +\bigwedge_{i=1}^{n} p_{i j} g_{i}\left(x_{i}\left(t-\sigma_{i j}(t)\right)\right) \\
& +\bigwedge_{i=1}^{n} F_{i j} \nu_{i}+\bigvee_{i=1}^{n} q_{i j} g_{i}\left(x_{i}\left(t-\sigma_{i j}(t)\right)\right) \\
& +\bigvee_{i=1}^{n} G_{i j} \nu_{i}+J_{j}, \quad t \in \mathbb{T}, j=1,2, \ldots, m,
\end{aligned}
$$

where $\mathbb{T}$ is a time scale. Though the nonimpulsive systems have been well studied in theory and in practice, the theory of impulsive differential equations is now being recognized to be richer than the corresponding theory of differential equations without impulses (see [33-35]). What is more, very few results are available on exponential stability of equilibrium point for fuzzy BAM neural networks with time-varying delays in leakage terms and impulses.
Motivated by the above discussion, in this paper, we consider the following model:

$$
\begin{aligned}
& x_{i}^{\prime}(t)=-a_{i}(t) x_{i}\left(t-\alpha_{i}(t)\right)+\sum_{j=1}^{m} a_{i j}(t) f_{j}\left(y_{j}(t)\right) \\
& +\sum_{j=1}^{m} b_{i j}(t) f_{j}\left(y_{j}(t-\tau(t))\right)+\sum_{j=1}^{m} c_{i j}(t) \omega_{j} \\
& +\bigwedge_{j=1}^{m} \alpha_{i j}(t) \int_{-\infty}^{t} k_{j}(t-s) f_{j}\left(y_{j}(s)\right) d s \\
& +\bigvee_{j=1}^{m} \beta_{i j}(t) \int_{-\infty}^{t} k_{j}(t-s) f_{j}\left(y_{j}(s)\right) d s \\
& +\bigwedge_{j=1}^{m} T_{i j} \omega_{j}+\bigvee_{j=1}^{m} H_{i j} \omega_{j}+A_{i}(t) \\
& t \geq 0, \quad t \neq t_{k}, \quad i=1,2, \ldots, n, \\
& \Delta x_{i}\left(t_{k}\right)=I_{k}\left(x_{i}\left(t_{k}\right)\right), \quad i=1,2, \ldots, n, k=1,2, \ldots, \\
& y_{j}^{\prime}(t)=-b_{j}(t) y_{j}\left(t-\beta_{j}(t)\right)+\sum_{i=1}^{n} d_{j i}(t) g_{i}\left(x_{i}(t)\right) \\
& +\sum_{i=1}^{n} p_{j i}(t) g_{i}\left(x_{i}(t-\rho(t))\right)+\sum_{i=1}^{n} q_{j i}(t) \mu_{i} \\
& +\bigwedge_{i=1}^{n} \gamma_{j i}(t) \int_{-\infty}^{t} k_{i}(t-s) g_{i}\left(x_{i}(s)\right) d s \\
& +\bigvee_{i=1}^{n} \eta_{j i}(t) \int_{-\infty}^{t} k_{i}(t-s) g_{i}\left(x_{i}(s)\right) d s \\
& +\bigwedge_{i=1}^{n} R_{j i} \mu_{i}+\bigvee_{i=1}^{n} S_{j i} \mu_{i}+B_{j}(t), \\
& t \geq 0, \quad t \neq t_{k}, \quad j=1,2, \ldots, m, \\
& \Delta y_{j}\left(t_{k}\right)=J_{k}\left(y_{j}\left(t_{k}\right)\right), \quad j=1,2, \ldots, m, k=1,2, \ldots,
\end{aligned}
$$

where $x_{i}(t)$ and $y_{j}(t)$ are the states of the $i$ th neuron and the $j$ th neuron at time $t, g_{i}(t)$ and $f_{j}(t)$ denote the activation functions of the $i$ th neuron and the $j$ th neuron at time $t, \mu_{i}$ and $\omega_{j}$ denote the inputs of the $i$ th neuron and the $j$ th neuron, $A_{i}(t)$ and $B_{j}(t)$ denote the bias of the $i$ th neuron and the $j$ th neuron at time $t, a_{i}(t)$ and $b_{j}(t)$ represent the rates with which the $i$ th neuron and the $j$ th neuron at time $t$ will reset their potential to the resting state in isolation when disconnected from the networks and external inputs, $a_{i j}(t), b_{i j}(t), d_{j i}(t)$, and $p_{j i}(t)$ denote the connection weights of the feedback template at time $t$ and $c_{i j}(t), q_{j i}(t)$ denote the connection weights of the 
feedforward template at time $t, \alpha_{i j}(t), \gamma_{j i}(t)$ and $\beta_{i j}(t), \eta_{j i}(t)$ denote the connection weights of the delays fuzzy feedback MIN template at time $t$ and the delays fuzzy feedback MAX template at time $t, T_{i j}, R_{j i}$ and $H_{i j}, S_{j i}$ are the elements of the fuzzy feedforward MIN template and fuzzy feedforward MAX template, $\bigwedge$ and $\bigvee$ denote the fuzzy AND and fuzzy OR operators, $0 \leq \tau(t) \leq \tau$ and $0 \leq \rho(t) \leq \rho$ denote the transmission delays at time $t, \Delta x_{i}\left(t_{k}\right)=x_{i}\left(t_{k}^{+}\right)-x_{i}\left(t_{k}\right)$, $\Delta y_{j}\left(t_{k}\right)=y_{j}\left(t_{k}^{+}\right)-y_{j}\left(t_{k}\right)$ are the impulses at moments $t_{k}$, and $t_{1}<t_{2}<\cdots$ is a strictly increasing sequence such that $\lim _{k \rightarrow \infty} t_{k}=+\infty, k_{j}(s) \geq 0$, and $k_{i}(s) \geq 0$ are the feedback kernels and satisfy $\int_{0}^{+\infty} k_{j}(s) d s=1, \int_{0}^{+\infty} k_{i}(s) d s=1, i=$ $1,2, \ldots, n, j=1,2, \ldots, m$.

The initial conditions are given by

$$
\begin{array}{ll}
x_{i}(s)=\varphi_{i}(s), & s \in(-\infty, 0], \\
y_{j}(s)=\psi_{j}(s), & s \in(-\infty, 0],
\end{array}
$$

where $\varphi_{i}, \psi_{j} \in C((-\infty, 0], R), i=1,2, \ldots, n, j=1,2, \ldots, m$.

For convenience, for a continuous function $f: R \rightarrow R$, we denote $f^{+}=\sup _{t \in R}|f(t)|, f^{-}=\inf _{t \in R}|f(t)|$. tions.

Throughout this paper, we make the following assump-

$\left(\mathrm{H}_{1}\right)$ The neuron activation functions $f_{j}(t)$ and $g_{i}(t), i=$ $1,2, \ldots, n, j=1,2, \ldots, m$, are continuous on $R$ and there exist some real constants $L_{j}, M_{i}$ such that

$$
\begin{gathered}
\left|f_{j}(u)-f_{j}(v)\right| \leq L_{j}|u-v|, \\
\left|g_{i}(u)-g_{i}(v)\right| \leq M_{i}|u-v|
\end{gathered}
$$

for all $u, v \in R, i=1,2, \ldots, n, j=1,2, \ldots, m$.

$\left(\mathrm{H}_{2}\right)$ The leakage delays satisfy $\alpha_{i}(t) \geq 0, \beta_{j}(t) \geq 0$ and $a_{i}(t)>0, b_{j}(t)>0, i=1,2, \ldots, n, j=1,2, \ldots, m$.

Definition 1. Let $z^{*}(t)=\left(x_{1}^{*}(t), x_{2}^{*}(t), \ldots, x_{n}^{*}(t), y_{1}^{*}(t)\right.$, $\left.y_{2}^{*}(t), \ldots, y_{m}^{*}(t)\right)^{T}$ be a solution of system (3) with the initial condition $\phi^{*}(t)=\left(\varphi_{1}^{*}(t), \varphi_{2}^{*}(t), \ldots, \varphi_{n}^{*}(t)\right.$, $\left.\psi_{1}^{*}(t), \psi_{2}^{*}(t), \ldots, \psi_{m}^{*}(t)\right)^{T}$ and let $z(t)=\left(x_{1}(t), x_{2}(t), \ldots\right.$, $\left.x_{n}(t), y_{1}(t), y_{2}(t), \ldots, y_{m}(t)\right)^{T}$ be any solution of system (3) with the initial condition $\phi(t)=\left(\varphi_{1}(t), \varphi_{2}(t), \ldots\right.$, $\left.\varphi_{n}(t), \psi_{1}(t), \psi_{2}(t), \ldots, \psi_{m}(t)\right)^{T}$; if these two solutions satisfy

$$
\begin{array}{r}
x_{i}(t)-x_{i}^{*}(t)=O\left(e^{-\lambda t}\right), \quad y_{j}(t)-y_{j}^{*}(t)=O\left(e^{-\lambda t}\right), \\
i=1,2, \ldots, n, \quad j=1,2, \ldots, m,
\end{array}
$$

then, we say that system (3) is exponentially stable.
Lemma 2 (see [36]). Let $z, z^{\prime}$ be two states of system (3); for $i=1,2, \ldots, n, j=1,2, \ldots, m$, one has

$$
\begin{aligned}
& \left|\bigwedge_{j=1}^{m} \alpha_{i j}(t) f_{j}(z)-\bigwedge_{j=1}^{m} \alpha_{i j}(t) f_{j}\left(z^{\prime}\right)\right| \\
& \quad \leq \sum_{j=1}^{m}\left|\alpha_{i j}(t)\right|\left|f_{j}(z)-f_{j}\left(z^{\prime}\right)\right| \\
& \left|\bigvee_{j=1}^{m} \beta_{i j}(t) f_{j}(z)-\bigvee_{j=1}^{m} \beta_{i j}(t) f_{j}\left(z^{\prime}\right)\right| \\
& \leq \sum_{j=1}^{m}\left|\beta_{i j}(t)\right|\left|f_{j}(z)-f_{j}\left(z^{\prime}\right)\right| \\
& \left|\bigwedge_{i=1}^{n} \gamma_{j i}(t) g_{i}(z)-\bigwedge_{i=1}^{n} \gamma_{j i}(t) g_{i}\left(z^{\prime}\right)\right| \\
& \leq \sum_{i=1}^{n}\left|\gamma_{j i}(t)\right|\left|g_{i}(z)-g_{i}\left(z^{\prime}\right)\right| \\
& \left|\bigvee_{j=1}^{n} \eta_{j i}(t) g_{i}(z)-\bigvee_{i=1}^{n} \eta_{j i}(t) g_{i}\left(z^{\prime}\right)\right| \\
& \eta_{j i}(t)|| g_{i}(z)-g_{i}\left(z^{\prime}\right) \mid
\end{aligned}
$$

Our main purpose of this paper is by using differential inequality techniques to study the exponential stability of (3). The results of this paper are completely new and complementary to the previously known results and the methods used in this paper are different from those used in $[31,32]$.

\section{Exponential Stability}

In this section, we will give some sufficient conditions to guarantee the exponential stability of system (3).

Theorem 3. Suppose that $\left(H_{1}\right)$ and $\left(H_{2}\right)$ hold. Let $z^{*}(t)=\left(x_{1}^{*}(t), x_{2}^{*}(t), \ldots, x_{n}^{*}(t), y_{1}^{*}(t), y_{2}^{*}(t), \ldots, y_{m}^{*}(t)\right)^{T}$ be a solution of system (3) with the initial condition $\phi^{*}(t)=\left(\varphi_{1}^{*}(t), \varphi_{2}^{*}(t), \ldots, \varphi_{n}^{*}(t), \psi_{1}^{*}(t), \psi_{2}^{*}(t), \ldots, \psi_{m}^{*}(t)\right)^{T}$. Furthermore, assume that

$\left(\mathrm{H}_{3}\right)$

$$
\begin{gathered}
-\left[a_{i}(t)-a_{i}(t) \alpha_{i}(t) a_{i}^{+}\right]+\left(1+a_{i}(t) \alpha_{i}(t)\right) \\
\times \sum_{j=1}^{m}\left(a_{i j}^{+}+b_{i j}^{+}+\alpha_{i j}^{+}+\beta_{i j}^{+}\right) L_{j}<0,
\end{gathered}
$$

$$
i=1,2, \ldots, n,
$$




$$
\begin{gathered}
-\left[b_{j}(t)-b_{j}(t) \beta_{j}(t) b_{j}^{+}\right]+\left(1+b_{j}(t) \beta_{j}(t)\right) \\
\times \sum_{i=1}^{n}\left(d_{j i}^{+}+p_{j i}^{+}+\gamma_{j i}^{+}+\eta_{j i}^{+}\right) M_{i}<0 \\
j=1,2, \ldots, m .
\end{gathered}
$$

$\left(\mathrm{H}_{4}\right)$ There exist constants $\theta_{i k}, \bar{\theta}_{j k}$ such that

$$
\begin{array}{r}
I_{k}\left(x_{i}\left(t_{k}\right)\right)=-\theta_{i k} x_{i}\left(t_{k}\right), \\
0 \leq \theta_{i k} \leq 2, \quad i=1,2, \ldots, n, \quad k=1,2, \ldots, \\
J_{k}\left(y_{j}\left(t_{k}\right)\right)=-\bar{\theta}_{j k} y_{j}\left(t_{k}\right), \\
0 \leq \bar{\theta}_{j k} \leq 2, \quad j=1,2, \ldots, m, \quad k=1,2, \ldots
\end{array}
$$

$$
\begin{aligned}
& \bar{y}_{j}^{\prime}(t)=-b_{j}(t) \bar{y}_{j}\left(t-\beta_{j}(t)\right)+\sum_{i=1}^{n} d_{j i}(t) \tilde{g}_{i}\left(\bar{x}_{i}(t)\right) \\
&+\sum_{i=1}^{n} p_{j i}(t) \tilde{g}_{i}\left(\bar{x}_{i}(t-\rho(t))\right) \\
&+\bigwedge_{i=1}^{n} \gamma_{j i}(t) \int_{-\infty}^{t} k_{i}(t-s) \tilde{g}_{i}\left(\bar{x}_{i}(s)\right) d s \\
&+\bigvee_{i=1}^{n} \eta_{j i}(t) \int_{-\infty}^{t} k_{i}(t-s) \tilde{g}_{i}\left(\bar{x}_{i}(s)\right) d s, \\
& j=1,2, \ldots, m .
\end{aligned}
$$

\section{According to $\left(\mathrm{H}_{4}\right)$, we get}

Then system (3) is exponentially stable.

Proof. Let $z(t)=\left(x_{1}(t), x_{2}(t), \ldots, x_{n}(t), y_{1}(t), y_{2}(t), \ldots\right.$, $\left.y_{m}(t)\right)^{T}$ be an arbitrary solution of system (3) with the initial condition $\phi(t)=\left(\varphi_{1}(t), \varphi_{2}(t), \ldots, \varphi_{n}(t), \psi_{1}(t), \psi_{2}(t), \ldots\right.$, $\left.\psi_{m}(t)\right)^{T}$. Set

$$
\begin{gathered}
\bar{x}_{i}(t)=x_{i}(t)-x_{i}^{*}(t), \quad i=1,2, \ldots, n, \\
\bar{y}_{j}(t)=y_{j}(t)-y_{j}^{*}(t), \quad j=1,2, \ldots, m, \\
\tilde{f}_{j}\left(\bar{y}_{j}(t)\right)=f_{j}\left(\bar{y}_{j}(t)+y_{j}^{*}(t)\right)-f_{j}\left(y_{j}^{*}(t)\right), \\
j=1,2, \ldots, m, \\
\tilde{g}_{i}\left(\bar{x}_{i}(t)\right)=g_{i}\left(\bar{x}_{i}(t)+x_{i}^{*}(t)\right)-g_{i}\left(x_{i}^{*}(t)\right), \\
i=1,2, \ldots, n .
\end{gathered}
$$

$$
\begin{aligned}
& x_{i}\left(t_{k}^{+}\right)-x_{i}^{*}\left(t_{k}^{+}\right) \\
&= x_{i}\left(t_{k}\right)+I_{k}\left(x_{i}\left(t_{k}\right)\right)-x_{i}^{*}\left(t_{k}\right)-I_{k}\left(x_{i}^{*}\left(t_{k}\right)\right) \\
&=\left(1-\theta_{i k}\right)\left(x_{i}\left(t_{k}\right)-x_{i}^{*}\left(t_{k}\right)\right), \\
& i=1,2, \ldots, n, \quad k=1,2, \ldots, \\
& y_{j}\left(t_{k}^{+}\right)-y_{j}^{*}\left(t_{k}^{+}\right) \\
&=y_{j}\left(t_{k}\right)+J_{k}\left(y_{j}\left(t_{k}\right)\right)-y_{j}^{*}\left(t_{k}\right)-J_{k}\left(y_{j}^{*}\left(t_{k}\right)\right) \\
&=\left(1-\bar{\theta}_{j k}\right)\left(y_{j}\left(t_{k}\right)-y_{j}^{*}\left(t_{k}\right)\right), \\
& j=1,2, \ldots, m, \quad k=1,2, \ldots
\end{aligned}
$$

So,

From (3) and (11), for $t>0, t \neq t_{k}, k=1,2, \ldots$, we have

$$
\begin{aligned}
\bar{x}_{i}^{\prime}(t)= & -a_{i}(t) \bar{x}_{i}\left(t-\alpha_{i}(t)\right)+\sum_{j=1}^{m} a_{i j}(t) \tilde{f}_{j}\left(\bar{y}_{j}(t)\right) \\
& +\sum_{j=1}^{m} b_{i j}(t) \tilde{f}_{j}\left(\bar{y}_{j}(t-\tau(t))\right) \\
& +\bigwedge_{j=1}^{m} \alpha_{i j}(t) \int_{-\infty}^{t} k_{j}(t-s) \tilde{f}_{j}\left(\bar{y}_{j}(s)\right) d s
\end{aligned}
$$


which implies that

$$
\begin{aligned}
\left|\bar{x}_{i}\left(t_{k}^{+}\right)\right|= & \left|x_{i}\left(t_{k}^{+}\right)-x_{i}^{*}\left(t_{k}^{+}\right)\right| \\
\leq & \left|x_{i}\left(t_{k}\right)-x_{i}^{*}\left(t_{k}\right)\right|=\left|\bar{x}_{i}\left(t_{k}^{-}\right)\right|, \\
& i=1,2, \ldots, n, \quad k=1,2, \ldots, \\
\left|\bar{y}_{j}\left(t_{k}^{+}\right)\right|= & \left|y_{j}\left(t_{k}^{+}\right)-y_{j}^{*}\left(t_{k}^{+}\right)\right| \\
\leq & \left|y_{j}\left(t_{k}\right)-y_{j}^{*}\left(t_{k}\right)\right|=\left|\bar{y}_{j}\left(t_{k}^{-}\right)\right|, \\
& j=1,2, \ldots, m, \quad k=1,2, \ldots .
\end{aligned}
$$

Define continuous functions $\Gamma_{i}(\omega)$ and $\Gamma_{j}(\omega)$ by setting

$$
\begin{aligned}
& \Gamma_{i}(\omega) \\
& \begin{aligned}
= & {\left[a_{i}(t) e^{\omega \alpha_{i}(t)}-\omega-a_{i}(t) e^{\omega \alpha_{i}(t)} \alpha_{i}(t)\right.} \\
& \left.\times\left(\omega+a_{i}^{+} e^{\omega \alpha_{i}^{+}}\right)\right]+\left[1+a_{i}(t) e^{\omega \alpha_{i}(t)} \alpha_{i}(t)\right] \\
& \times \sum_{j=1}^{m}\left[a_{i j}^{+}+b_{i j}^{+} e^{\omega \tau}+\left(\alpha_{i j}^{+}+\beta_{i j}^{+}\right) \int_{0}^{+\infty} k_{j}(u) e^{\omega u} d u\right] L_{j}, \\
\Gamma_{j}(\omega) & i=1,2, \ldots, n, \\
=- & {\left[b_{j}(t) e^{\omega \beta_{j}(t)}-\omega-b_{j}(t) e^{\omega \beta_{j}(t)} \beta_{j}(t)\right.} \\
& \left.\times\left(\omega+b_{j}^{+} e^{\omega \beta_{j}^{+}}\right)\right]+\left[1+b_{j}(t) e^{\omega \beta_{j}(t)} \beta_{j}(t)\right] \\
& \times \sum_{i=1}^{n}\left[d_{j i}^{+}+p_{j i}^{+} e^{\omega \rho}+\left(\gamma_{j i}^{+}+\eta_{j i}^{+}\right) \int_{0}^{+\infty} k_{i}(u) e^{\omega u} d u\right] M_{i},
\end{aligned} \\
&
\end{aligned}
$$

Then, for $i=1,2, \ldots, n, j=1,2, \ldots, m$, we have

$$
\begin{aligned}
\Gamma_{i}(0)= & -\left[a_{i}(t)-a_{i}(t) \alpha_{i}(t) a_{i}^{+}\right]+\left(1+a_{i}(t) \alpha_{i}(t)\right) \\
& \times \sum_{j=1}^{m}\left(a_{i j}^{+}+b_{i j}^{+}+\alpha_{i j}^{+}+\beta_{i j}^{+}\right) L_{j}<0, \\
\Gamma_{j}(0)= & -\left[b_{j}(t)-b_{j}(t) \beta_{j}(t) b_{j}^{+}\right]+\left(1+b_{j}(t) \beta_{j}(t)\right) \\
& \times \sum_{i=1}^{n}\left(d_{j i}^{+}+p_{j i}^{+}+\gamma_{j i}^{+}+\eta_{j i}^{+}\right) M_{i}<0 .
\end{aligned}
$$

The continuity of $\Gamma_{i}(\omega)$ and $\Gamma_{j}(\omega)$ implies that there exists $\lambda>$ 0 such that

$$
\begin{aligned}
& \Gamma_{i}(\lambda)=-\left[a_{i}(t) e^{\lambda \alpha_{i}(t)}-\lambda-a_{i}(t) e^{\lambda \alpha_{i}(t)} \alpha_{i}(t)\right. \\
& \left.\times\left(\lambda+a_{i}^{+} e^{\lambda \alpha_{i}^{+}}\right)\right]+\left[1+a_{i}(t) e^{\lambda \alpha_{i}(t)} \alpha_{i}(t)\right] \\
& \times \sum_{j=1}^{m}\left[a_{i j}^{+}+b_{i j}^{+} e^{\lambda \tau}+\left(\alpha_{i j}^{+}+\beta_{i j}^{+}\right) \int_{0}^{+\infty} k_{j}(u) e^{\lambda u} d u\right] \\
& \times L_{j}<0, \quad i=1,2, \ldots, n, \\
& \Gamma_{j}(\lambda)=-\left[b_{j}(t) e^{\lambda \beta_{j}(t)}-\lambda-b_{j}(t) e^{\lambda \beta_{j}(t)} \beta_{j}(t)\right. \\
& \left.\times\left(\lambda+b_{j}^{+} e^{\lambda \beta_{j}^{+}}\right)\right]+\left[1+b_{j}(t) e^{\lambda \beta_{j}(t)} \beta_{j}(t)\right] \\
& \times \sum_{i=1}^{n}\left[d_{j i}^{+}+p_{j i}^{+} e^{\lambda \rho}+\left(\gamma_{j i}^{+}+\eta_{j i}^{+}\right) \int_{0}^{+\infty} k_{i}(u) e^{\lambda u} d u\right] \\
& \times M_{i}<0, \quad j=1,2, \ldots, m . \\
& \text { Let } \\
& X_{i}(t)=\bar{x}_{i}(t) e^{\lambda t}, \quad Y_{j}(t)=\bar{y}_{j}(t) e^{\lambda t}, \\
& i=1,2, \ldots, n, \quad j=1,2, \ldots, m .
\end{aligned}
$$

From (12) and (20), we obtain

$$
\begin{aligned}
X_{i}^{\prime}(t)= & \lambda e^{\lambda t} \bar{x}_{i}(t)+e^{\lambda t} \bar{x}_{i}^{\prime}(t) \\
= & \lambda X_{i}(t)+e^{\lambda t} \\
\times & {\left[-a_{i}(t) \bar{x}_{i}\left(t-\alpha_{i}(t)\right)+\sum_{j=1}^{m} a_{i j}(t) \tilde{f}_{j}\left(\bar{y}_{j}(t)\right)\right.} \\
& +\sum_{j=1}^{m} b_{i j}(t) \tilde{f}_{j}\left(\bar{y}_{j}(t-\tau(t))\right) \\
& +\bigwedge_{j=1}^{m} \alpha_{i j}(t) \int_{-\infty}^{t} k_{j}(t-s) \tilde{f}_{j}\left(\bar{y}_{j}(s)\right) d s \\
& \left.+\bigvee_{j=1}^{m} \beta_{i j}(t) \int_{-\infty}^{t} k_{j}(t-s) \tilde{f}_{j}\left(\bar{y}_{j}(s)\right) d s\right]
\end{aligned}
$$




$$
\begin{aligned}
= & \lambda X_{i}(t)-a_{i}(t) e^{\lambda \alpha_{i}(t)} X_{i}\left(t-\alpha_{i}(t)\right) \\
& +\sum_{j=1}^{m} a_{i j}(t) e^{\lambda t} \tilde{f}_{j}\left(\bar{y}_{j}(t)\right) \\
& +\sum_{j=1}^{m} b_{i j}(t) e^{\lambda t} \tilde{f}_{j}\left(\bar{y}_{j}(t-\tau(t))\right) \\
& +\bigwedge_{j=1}^{m} \alpha_{i j}(t) e^{\lambda t} \int_{-\infty}^{t} k_{j}(t-s) \tilde{f}_{j}\left(\bar{y}_{j}(s)\right) d s \\
& +\bigvee_{j=1}^{m} \beta_{i j}(t) e^{\lambda t} \int_{-\infty}^{t} k_{j}(t-s) \tilde{f}_{j}\left(\bar{y}_{j}(s)\right) d s, \\
& i=1,2, \ldots, n .
\end{aligned}
$$

Similarly,

$$
\begin{aligned}
& Y_{j}^{\prime}(t)= \lambda Y_{j}(t)-b_{j}(t) e^{\lambda \beta_{j}(t)} Y_{j}\left(t-\beta_{j}(t)\right) \\
&+\sum_{i=1}^{n} d_{j i}(t) e^{\lambda t} \widetilde{g}_{i}\left(\bar{x}_{i}(t)\right) \\
&+\sum_{i=1}^{n} p_{j i}(t) e^{\lambda t} \widetilde{g}_{i}\left(\bar{x}_{i}(t-\rho(t))\right) \\
&+\bigwedge_{i=1}^{n} \gamma_{j i}(t) e^{\lambda t} \int_{-\infty}^{t} k_{i}(t-s) \widetilde{g}_{i}\left(\bar{x}_{i}(s)\right) d s \\
&+\bigvee_{i=1}^{n} \eta_{j i}(t) e^{\lambda t} \int_{-\infty}^{t} k_{i}(t-s) \tilde{g}_{i}\left(\bar{x}_{i}(s)\right) d s, \\
& j=1,2, \ldots, m .
\end{aligned}
$$

We rewrite (21) and (22) as follows:

$$
\begin{aligned}
& X_{i}^{\prime}(t) \\
& \begin{aligned}
=\lambda X_{i}(t)- & a_{i}(t) e^{\lambda \alpha_{i}(t)} X_{i}(t)+a_{i}(t) e^{\lambda \alpha_{i}(t)} \\
\times \int_{t-\alpha_{i}(t)}^{t}\{ & \lambda X_{\mathrm{i}}(s)-a_{i}(s) e^{\lambda \alpha_{i}(s)} \times X_{i}\left(s-\alpha_{i}(s)\right) \\
& +\sum_{j=1}^{m} a_{i j}(s) e^{\lambda s} \tilde{f}_{j}\left(\bar{y}_{j}(s)\right) \\
& +\sum_{j=1}^{m} b_{i j}(s) e^{\lambda s} \tilde{f}_{j}\left(\bar{y}_{j}(s-\tau(s))\right) \\
& +\bigwedge_{j=1}^{m} \alpha_{i j}(s) e^{\lambda s} \int_{-\infty}^{s} k_{j}(s-u) \tilde{f}_{j}\left(\bar{y}_{j}(u)\right) d u
\end{aligned}
\end{aligned}
$$

(21)

$$
\begin{aligned}
& Y_{j}^{\prime}(t) \\
& =\lambda Y_{j}(t)-b_{j}(t) e^{\lambda \beta_{j}(t)} Y_{j}(t)+b_{j}(t) e^{\lambda \beta_{j}(t)} \\
& \times \int_{t-\beta_{j}(t)}^{t}\left\{\lambda Y_{j}(s)-b_{j}(s) e^{\lambda \beta_{j}(s)}\right. \\
& \quad \times Y_{j}\left(s-\beta_{j}(s)\right)+\sum_{i=1}^{n} d_{j i}(s) e^{\lambda s} \widetilde{g}_{i}\left(\bar{x}_{i}(s)\right) \\
& +\sum_{i=1}^{n} p_{j i}(s) e^{\lambda s} \widetilde{g}_{i}\left(\bar{x}_{i}(s-\rho(s))\right) \\
& +\bigwedge_{i=1}^{n} \gamma_{j i}(s) e^{\lambda s} \int_{-\infty}^{s} k_{i}(s-u) \widetilde{g}_{i}\left(\bar{x}_{i}(u)\right) d u \\
& \left.+\bigvee_{i=1}^{n} \eta_{j i}(s) e^{\lambda s} \int_{-\infty}^{s} k_{i}(s-u) \widetilde{g}_{i}\left(\bar{x}_{i}(u)\right) d u\right\} d s
\end{aligned}
$$$$
+\sum_{i=1}^{n} d_{j i}(t) e^{\lambda t} \widetilde{g}_{i}\left(\bar{x}_{i}(t)\right)
$$$$
+\sum_{i=1}^{n} p_{j i}(t) e^{\lambda t} \widetilde{g}_{i}\left(\bar{x}_{i}(t-\rho(t))\right)
$$$$
+\bigwedge_{i=1}^{n} \gamma_{j i}(t) e^{\lambda t} \int_{-\infty}^{t} k_{i}(t-s) \widetilde{g}_{i}\left(\bar{x}_{i}(s)\right) d s
$$$$
+\bigvee_{i=1}^{n} \eta_{j i}(t) e^{\lambda t} \int_{-\infty}^{t} k_{i}(t-s) \tilde{g}_{i}\left(\bar{x}_{i}(s)\right) d s,
$$

$$
j=1,2, \ldots, m \text {. }
$$


We define a positive number such that

$$
M=\max _{\substack{1 \leq i \leq n, 1 \leq j \leq m}}\left\{\sup _{s \in[-\tau, 0]}\left|X_{i}(s)\right|, \sup _{s \in[-\rho, 0]}\left|Y_{j}(s)\right|\right\}
$$

It follows that

$$
\begin{gathered}
\left|X_{i}(t)\right|<M, \quad \forall t \in[-\tau, 0], i=1,2, \ldots, n, \\
\left|Y_{j}(t)\right|<M, \quad \forall t \in[-\rho, 0], \quad j=1,2, \ldots, m .
\end{gathered}
$$

We claim that

$$
\left|X_{i}(t)\right|<M, \quad\left|Y_{j}(t)\right|<M,
$$

$\forall t>0, \quad t \neq t_{k}, \quad i=1,2, \ldots, n, \quad j=1,2, \ldots, m$.

If (27) is not valid, then there exist some $i \in\{1,2, \ldots, n\}$, some $j \in\{1,2, \ldots, m\}$, and a first $T_{1}>0$ such that one of the following four cases must occur:

(1) $X_{i}\left(T_{1}\right)=M, X_{i}^{\prime}\left(T_{1}\right) \geq 0,\left|X_{i}(t)\right|<M,\left|Y_{j}(t)\right|<M$, for $t<T_{1}$

(2) $X_{i}\left(T_{1}\right)=-M, X_{i}^{\prime}\left(T_{1}\right) \leq 0,\left|X_{i}(t)\right|<M,\left|Y_{j}(t)\right|<M$, for $t<T_{1}$;

(3) $Y_{j}\left(T_{1}\right)=M, Y_{j}^{\prime}\left(T_{1}\right) \geq 0,\left|X_{i}(t)\right|<M,\left|Y_{j}(t)\right|<M$, for $t<T_{1}$;

(4) $Y_{j}\left(T_{1}\right)=-M, Y_{j}^{\prime}\left(T_{1}\right) \leq 0,\left|X_{i}(t)\right|<M,\left|Y_{j}(t)\right|<M$, for $t<T_{1}$.

If (1) holds from (19), (23), and $\left(\mathrm{H}_{3}\right)$, we have

$$
\begin{aligned}
& X_{i}^{\prime}\left(T_{1}\right) \\
& =\lambda X_{i}\left(T_{1}\right)-a_{i}\left(T_{1}\right) e^{\lambda \alpha_{i}\left(T_{1}\right)} X_{i}\left(T_{1}\right)+a_{i}\left(T_{1}\right) e^{\lambda \alpha_{i}\left(T_{1}\right)} \\
& \quad \times \int_{T_{1}-\alpha_{i}\left(T_{1}\right)}^{T_{1}}\left\{\lambda X_{i}(s)-a_{i}(s) e^{\lambda \alpha_{i}(s)} X_{i}\left(s-\alpha_{i}(s)\right)\right. \\
& +\sum_{j=1}^{m} a_{i j}(s) e^{\lambda s} \widetilde{f}_{j}\left(\bar{y}_{j}(s)\right) \\
& +\sum_{j=1}^{m} b_{i j}(s) e^{\lambda s} \widetilde{f}_{j}\left(\bar{y}_{j}(s-\tau(s))\right)
\end{aligned}
$$

$$
\begin{aligned}
& +\bigwedge_{j=1}^{m} \alpha_{i j}(s) e^{\lambda s} \int_{-\infty}^{s} k_{j}(s-u) \tilde{f}_{j}\left(\bar{y}_{j}(u)\right) d u \\
& +\bigvee_{j=1}^{m} \beta_{i j}(s) e^{\lambda s} \\
& \left.\times \int_{-\infty}^{s} k_{j}(s-u) \tilde{f}_{j}\left(\bar{y}_{j}(u)\right) d u\right\} d s
\end{aligned}
$$$$
+\sum_{j=1}^{m} a_{i j}\left(T_{1}\right) e^{\lambda T_{1}} \widetilde{f}_{j}\left(\bar{y}_{j}\left(T_{1}\right)\right)
$$$$
+\sum_{j=1}^{m} b_{i j}\left(T_{1}\right) e^{\lambda T_{1}} \widetilde{f}_{j}\left(\bar{y}_{j}(t-\tau(t))\right)
$$$$
+\bigwedge_{j=1}^{m} \alpha_{i j}\left(T_{1}\right) e^{\lambda t} \int_{-\infty}^{T_{1}} k_{j}\left(T_{1}-s\right) \tilde{f}_{j}\left(\bar{y}_{j}(s)\right) d s
$$$$
+\bigvee_{j=1}^{m} \beta_{i j}\left(T_{1}\right) e^{\lambda T_{1}} \int_{-\infty}^{T_{1}} k_{j}\left(T_{1}-s\right) \tilde{f}_{j}\left(\bar{y}_{j}(s)\right) d s
$$$$
\leq \lambda X_{i}\left(T_{1}\right)-a_{i}\left(T_{1}\right) e^{\lambda \alpha_{i}\left(T_{1}\right)} X_{i}\left(T_{1}\right)+a_{i}\left(T_{1}\right) e^{\lambda \alpha_{i}\left(T_{1}\right)}
$$$$
\times \int_{T_{1}-\alpha_{i}\left(T_{1}\right)}^{T_{1}}\left\{\lambda X_{i}\left(T_{1}\right)+a_{i}^{+} e^{\lambda \alpha_{i}^{+}} X_{i}\left(T_{1}\right)+\sum_{j=1}^{m} a_{i j}^{+} L_{j}\left|Y_{j}(s)\right|\right.
$$$$
+\sum_{j=1}^{m} b_{i j}^{+} e^{\lambda \tau} L_{j}\left|Y_{j}(s-\tau(s))\right|
$$$$
+\sum_{j=1}^{m} \alpha_{i j}^{+} \int_{-\infty}^{s} k_{j}(s-u) e^{\lambda(s-u)} L_{j}\left|Y_{j}(u)\right| d u
$$$$
+\sum_{j=1}^{m} \beta_{i j}^{+} \int_{-\infty}^{s} k_{j}(s-u) e^{\lambda(s-u)}
$$

$$
\left.\times L_{j}\left|Y_{j}(u)\right| d u\right\} d s
$$

$$
\begin{aligned}
+ & \sum_{j=1}^{m} a_{i j}^{+} L_{j}\left|Y_{j}\left(T_{1}\right)\right| \\
+ & \sum_{j=1}^{m} b_{i j}^{+} L_{j} e^{\lambda \tau}\left|Y_{j}\left(T_{1}-\tau\left(T_{1}\right)\right)\right| \\
+ & \sum_{j=1}^{m} \alpha_{i j}^{+} \int_{-\infty}^{T_{1}} k_{j}\left(T_{1}-s\right) e^{\lambda\left(T_{1}-s\right)} L_{j}\left|Y_{j}(s)\right| d s \\
+ & \sum_{j=1}^{m} \beta_{i j}^{+} \int_{-\infty}^{T_{1}} k_{j}\left(T_{1}-s\right) e^{\lambda\left(T_{1}-s\right)} L_{j}\left|Y_{j}(s)\right| d s \\
\leq & {\left[a_{i}\left(T_{1}\right) e^{\lambda \alpha_{i}\left(T_{1}\right)}-\lambda-a_{i}\left(T_{1}\right) e^{\lambda \alpha_{i}\left(T_{1}\right)}\right.} \\
& \left.\times \alpha_{i}\left(T_{1}\right)\left(\lambda+a_{i}^{+} e^{\lambda \alpha_{i}^{+}}\right)\right] X_{i}\left(T_{1}\right)+a_{i}\left(T_{1}\right) e^{\lambda \alpha_{i}\left(T_{1}\right)}
\end{aligned}
$$




$$
\begin{aligned}
& \times \int_{T_{1}-\alpha_{i}\left(T_{1}\right)}^{T_{1}}\left[\sum_{j=1}^{m} a_{i j}^{+} L_{j} M+\sum_{j=1}^{m} b_{i j}^{+} e^{\lambda \tau} L_{j} M+\sum_{j=1}^{m} \alpha_{i j}^{+} L_{j} M\right. \\
& \times \int_{-\infty}^{s} k_{j}(s-u) e^{\lambda(s-u)} d u \\
& \left.+\sum_{j=1}^{m} \beta_{i j}^{+} L_{j} M \int_{-\infty}^{s} k_{j}(s-u) e^{\lambda(s-u)} d u\right] d s \\
& +\sum_{j=1}^{m} a_{i j}^{+} L_{j} M+\sum_{j=1}^{m} b_{i j}^{+} e^{\lambda \tau} L_{j} M+\sum_{j=1}^{m} \alpha_{i j}^{+} L_{j} M \\
& \times \int_{-\infty}^{T_{1}} k_{j}\left(T_{1}-s\right) e^{\lambda\left(T_{1}-s\right)} d u \\
& +\sum_{j=1}^{m} \beta_{i j}^{+} L_{j} M \int_{-\infty}^{T_{1}} k_{j}\left(T_{1}-s\right) e^{\lambda\left(T_{1}-s\right)} d u \\
& \leq-\left[a_{i}\left(T_{1}\right) e^{\lambda \alpha_{i}\left(T_{1}\right)}-\lambda-a_{i}\left(T_{1}\right) e^{\lambda \alpha_{i}\left(T_{1}\right)}\right. \\
& \left.\times \alpha_{i}\left(T_{1}\right)\left(\lambda+a_{i}^{+} e^{\lambda \alpha_{i}^{+}}\right)\right] X_{i}\left(T_{1}\right) \\
& +\left(1+a_{i}\left(T_{1}\right) e^{\lambda \alpha_{i}\left(T_{1}\right)} \alpha_{i}\left(T_{1}\right)\right) \\
& \times \sum_{j=1}^{m}\left[a_{i j}^{+}+b_{i j}^{+} e^{\lambda \tau}+\left(\alpha_{i j}^{+}+\beta_{i j}^{+}\right) \times \int_{0}^{+\infty} k_{j}(u) e^{\lambda u} d u\right] L_{j} M \\
& =\left\{-\left[a_{i}\left(T_{1}\right) e^{\lambda \alpha_{i}\left(T_{1}\right)}-\lambda-a_{i}\left(T_{1}\right) e^{\lambda \alpha_{i}\left(T_{1}\right)}\right.\right. \\
& \left.\times \alpha_{i}\left(T_{1}\right)\left(\lambda+a_{i}^{+} e^{\lambda \alpha_{i}^{+}}\right)\right] \\
& +\left(1+a_{i}\left(T_{1}\right) e^{\lambda \alpha_{i}\left(T_{1}\right)} \alpha_{i}\left(T_{1}\right)\right) \\
& \times \sum_{j=1}^{m}\left[a_{i j}^{+}+b_{i j}^{+} e^{\lambda \tau}+\left(\alpha_{i j}^{+}+\beta_{i j}^{+}\right)\right. \\
& \left.\left.\times \int_{0}^{+\infty} k_{j}(u) e^{\lambda u} d u\right] L_{j}\right\} M
\end{aligned}
$$

$<0$,

and this is a contradiction. Hence, (1) does not hold.

If (2) holds, then, from $\left(\mathrm{H}_{3}\right),(19)$, and (24), we have

$$
\begin{aligned}
& X_{i}^{\prime}\left(T_{1}\right) \\
& =\lambda X_{i}\left(T_{1}\right)-a_{i}\left(T_{1}\right) e^{\lambda \alpha_{i}\left(T_{1}\right)} X_{i}\left(T_{1}\right)+a_{i}\left(T_{1}\right) e^{\lambda \alpha_{i}\left(T_{1}\right)} \\
& \times \int_{T_{1}-\alpha_{i}\left(T_{1}\right)}^{T_{1}}\left\{\lambda X_{i}(s)-a_{i}(s) \times e^{\lambda \alpha_{i}(s)} X_{i}\left(s-\alpha_{i}(s)\right)\right. \\
& +\sum_{j=1}^{m} a_{i j}(s) e^{\lambda s} \widetilde{f}_{j}\left(\bar{y}_{j}(s)\right)
\end{aligned}
$$$$
+\sum_{j=1}^{m} b_{i j}(s) e^{\lambda s} \tilde{f}_{j}\left(\bar{y}_{j}(s-\tau(s))\right)
$$$$
+\bigwedge_{j=1}^{m} \alpha_{i j}(s) e^{\lambda s} \int_{-\infty}^{s} k_{j}(s-u) \tilde{f}_{j}\left(\bar{y}_{j}(u)\right) d u
$$$$
+\bigvee_{j=1}^{m} \beta_{i j}(s) e^{\lambda s}
$$$$
\left.\times \int_{-\infty}^{s} k_{j}(s-u) \tilde{f}_{j}\left(\bar{y}_{j}(u)\right) d u\right\} d s
$$$$
+\sum_{j=1}^{m} a_{i j}\left(T_{1}\right) e^{\lambda T_{1}} \tilde{f}_{j}\left(\bar{y}_{j}\left(T_{1}\right)\right)
$$$$
+\sum_{j=1}^{m} b_{i j}\left(T_{1}\right) e^{\lambda T_{1}} \tilde{f}_{j}\left(\bar{y}_{j}(t-\tau(t))\right)
$$$$
+\bigwedge_{j=1}^{m} \alpha_{i j}\left(T_{1}\right) e^{\lambda t} \int_{-\infty}^{T_{1}} k_{j}\left(T_{1}-s\right) \tilde{f}_{j}\left(\bar{y}_{j}(s)\right) d s
$$$$
+\bigvee_{j=1}^{m} \beta_{i j}\left(T_{1}\right) e^{\lambda T_{1}} \int_{-\infty}^{T_{1}} k_{j}\left(T_{1}-s\right) \tilde{f}_{j}\left(\bar{y}_{j}(s)\right) d s
$$$$
\geq \lambda X_{i}\left(T_{1}\right)-a_{i}\left(T_{1}\right) e^{\lambda \alpha_{i}\left(T_{1}\right)} X_{i}\left(T_{1}\right)+a_{i}\left(T_{1}\right) e^{\lambda \alpha_{i}\left(T_{1}\right)}
$$$$
\times \int_{T_{1}-\alpha_{i}\left(T_{1}\right)}^{T_{1}}\left\{\lambda X_{i}\left(T_{1}\right)+a_{i}^{+} e^{\lambda \alpha_{i}^{+}} X_{i}\left(T_{1}\right)-\sum_{j=1}^{m} a_{i j}^{+} L_{j}\left|Y_{j}(s)\right|\right.
$$$$
-\sum_{j=1}^{m} b_{i j}^{+} e^{\lambda \tau} L_{j}\left|Y_{j}(s-\tau(s))\right|
$$$$
-\sum_{j=1}^{m} \alpha_{i j}^{+} \int_{-\infty}^{s} k_{j}(s-u) e^{\lambda(s-u)} L_{j}\left|Y_{j}(u)\right| d u
$$$$
-\sum_{j=1}^{m} \beta_{i j}^{+} \int_{-\infty}^{s} k_{j}(s-u) e^{\lambda(s-u)}
$$$$
\left.\times L_{j}\left|Y_{j}(u)\right| d u\right\} d s
$$

$$
\begin{aligned}
& -\sum_{j=1}^{m} a_{i j}^{+} L_{j}\left|Y_{j}\left(T_{1}\right)\right|-\sum_{j=1}^{m} b_{i j}^{+} L_{j} e^{\lambda \tau}\left|Y_{j}\left(T_{1}-\tau\left(T_{1}\right)\right)\right| \\
- & \sum_{j=1}^{m} \alpha_{i j}^{+} \int_{-\infty}^{T_{1}} k_{j}\left(T_{1}-s\right) e^{\lambda\left(T_{1}-s\right)} L_{j}\left|Y_{j}(s)\right| d s \\
- & \sum_{j=1}^{m} \beta_{i j}^{+} \int_{-\infty}^{T_{1}} k_{j}\left(T_{1}-s\right) e^{\lambda\left(T_{1}-s\right)} L_{j}\left|Y_{j}(s)\right| d s \\
\geq- & {\left[a_{i}\left(T_{1}\right) e^{\lambda \alpha_{i}\left(T_{1}\right)}-\lambda-a_{i}\left(T_{1}\right) e^{\lambda \alpha_{i}\left(T_{1}\right)}\right.} \\
& \left.\quad \times \alpha_{i}\left(T_{1}\right)\left(\lambda+a_{i}^{+} e^{\lambda \alpha_{i}^{+}}\right)\right] X_{i}\left(T_{1}\right)+a_{i}\left(T_{1}\right) e^{\lambda \alpha_{i}\left(T_{1}\right)}
\end{aligned}
$$




$$
\begin{aligned}
& \times \int_{T_{1}-\alpha_{i}\left(T_{1}\right)}^{T_{1}}\left[-\sum_{j=1}^{m} a_{i j}^{+} L_{j} M-\sum_{j=1}^{m} b_{i j}^{+} e^{\lambda \tau} L_{j} M-\sum_{j=1}^{m} \alpha_{i j}^{+} L_{j} M\right. \\
& \times \int_{-\infty}^{s} k_{j}(s-u) e^{\lambda(s-u)} d u \\
& \left.-\sum_{j=1}^{m} \beta_{i j}^{+} L_{j} M \int_{-\infty}^{s} k_{j}(s-u) e^{\lambda(s-u)} d u\right] d s \\
& -\sum_{j=1}^{m} a_{i j}^{+} L_{j} M-\sum_{j=1}^{m} b_{i j}^{+} e^{\lambda \tau} L_{j} M-\sum_{j=1}^{m} \alpha_{i j}^{+} L_{j} M \\
& \times \int_{-\infty}^{T_{1}} k_{j}\left(T_{1}-s\right) e^{\lambda\left(T_{1}-s\right)} d u \\
& -\sum_{j=1}^{m} \beta_{i j}^{+} L_{j} M \int_{-\infty}^{T_{1}} k_{j}\left(T_{1}-s\right) e^{\lambda\left(T_{1}-s\right)} d u \\
& \geq\left\{-\left[a_{i}\left(T_{1}\right) e^{\lambda \alpha_{i}\left(T_{1}\right)}-\lambda-a_{i}\left(T_{1}\right) e^{\lambda \alpha_{i}\left(T_{1}\right)}\right.\right. \\
& \left.\times \alpha_{i}\left(T_{1}\right)\left(\lambda+a_{i}^{+} e^{\lambda \alpha_{i}^{+}}\right)\right] X_{i}\left(T_{1}\right) \\
& +\left(1+a_{i}\left(T_{1}\right) e^{\lambda \alpha_{i}\left(T_{1}\right)} \alpha_{i}\left(T_{1}\right)\right) \\
& \times \sum_{j=1}^{m}\left[a_{i j}^{+}+b_{i j}^{+} e^{\lambda \tau}+\left(\alpha_{i j}^{+}+\beta_{i j}^{+}\right)\right. \\
& \left.\left.\times \int_{0}^{+\infty} k_{j}(u) e^{\lambda u} d u\right] L_{j}\right\}(-M)
\end{aligned}
$$

$>0$.

This is also a contradiction.

Similarly, if (3) (or (4)) holds, we can derive a contradiction. Therefore, (27) holds.

Furthermore, together with (16) and (17), we have

$$
\begin{aligned}
\left|\bar{x}_{i}\left(t_{k}^{+}\right)\right| & =\left|x_{i}\left(t_{k}^{+}\right)-x_{i}^{*}\left(t_{k}^{+}\right)\right| \\
& \leq\left|x_{i}\left(t_{k}\right)-x_{i}^{*}\left(t_{k}\right)\right|=\left|\bar{x}_{i}\left(t_{k}^{-}\right)\right| \\
& =\left|X_{i}\left(t_{k}^{-}\right)\right| e^{-\lambda t_{k}} \leq M e^{-\lambda t_{k}}, \\
\left|\bar{y}_{j}\left(t_{k}^{+}\right)\right| & =\left|y_{j}\left(t_{k}^{+}\right)-y_{j}^{*}\left(t_{k}^{+}\right)\right| \\
& \leq\left|y_{j}\left(t_{k}\right)-y_{j}^{*}\left(t_{k}\right)\right|=\left|\bar{y}_{j}\left(t_{k}^{-}\right)\right| \\
& =\left|Y_{j}\left(t_{k}^{-}\right)\right| e^{-\lambda t_{k}} \leq M e^{-\lambda t_{k}},
\end{aligned}
$$

where $i=1,2, \ldots, n, j=1,2, \ldots, m$, and $k=1,2, \ldots$.
From (27) and (30), we get

$$
\begin{aligned}
& x_{i}(t)-x_{i}^{*}(t)=O\left(e^{-\lambda t}\right), \\
& y_{j}(t)-y_{j}^{*}(t)=O\left(e^{-\lambda t}\right)
\end{aligned}
$$

for all $t>0, i=1,2, \ldots, n$, and $j=1,2, \ldots, m$. Therefore, system (3) is exponentially stable. This completes the proof.

\section{An Example}

In this section, we present an example to illustrate the feasibility of our results obtained in previous sections.

Example 4. Consider the following fuzzy BAM neural networks with distributed delays and impulses:

$$
\begin{aligned}
x_{i}^{\prime}(t)= & -a_{i}(t) x_{i}\left(t-\alpha_{i}(t)\right)+\sum_{j=1}^{2} a_{i j}(t) f_{j}\left(y_{j}(t)\right) \\
& +\sum_{j=1}^{2} b_{i j}(t) f_{j}\left(y_{j}(t-\tau(t))\right)+\sum_{j=1}^{2} c_{i j}(t) \omega_{j} \\
& +\bigwedge_{j=1}^{2} \alpha_{i j}(t) \int_{-\infty}^{t} k_{j}(t-s) f_{j}\left(y_{j}(s)\right) d s \\
& +\bigvee_{j=1}^{2} \beta_{i j}(t) \int_{-\infty}^{t} k_{j}(t-s) f_{j}\left(y_{j}(s)\right) d s \\
& +\bigwedge_{j=1}^{2} T_{i j} \omega_{j}+\bigvee_{j=1}^{m} H_{i j} \omega_{j}+A_{i}(t), \quad t \geq 0, \quad t \neq t_{k}, \\
& \Delta x_{i}\left(t_{k}\right)=-\theta_{i k} x_{i}\left(t_{k}\right), \quad k=1,2, \ldots,
\end{aligned}
$$

$$
\begin{aligned}
y_{j}^{\prime}(t)= & -b_{j}(t) y_{j}\left(t-\beta_{j}(t)\right)+\sum_{i=1}^{2} d_{j i}(t) g_{i}\left(x_{i}(t)\right) \\
& +\sum_{i=1}^{2} p_{j i}(t) g_{i}\left(x_{i}(t-\rho(t))\right) \\
& +\sum_{i=1}^{2} q_{j i}(t) \mu_{i}+\bigwedge_{i=1}^{n} \gamma_{j i}(t) \int_{-\infty}^{t} k_{i}(t-s) g_{i}\left(x_{i}(s)\right) d s \\
& +\bigvee_{i=1}^{2} \eta_{j i}(t) \int_{-\infty}^{t} k_{i}(t-s) g_{i}\left(x_{i}(s)\right) d s \\
& +\bigwedge_{i=1}^{2} R_{j i} \mu_{i}+\bigvee_{i=1}^{n} s_{j i} \mu_{i}+B_{j}(t), \quad t \geq 0, \quad t \neq t_{k}, \\
& \Delta y_{j}\left(t_{k}\right)=-\bar{\theta}_{j k} y_{j}\left(t_{k}\right), \quad k=1,2, \ldots,
\end{aligned}
$$




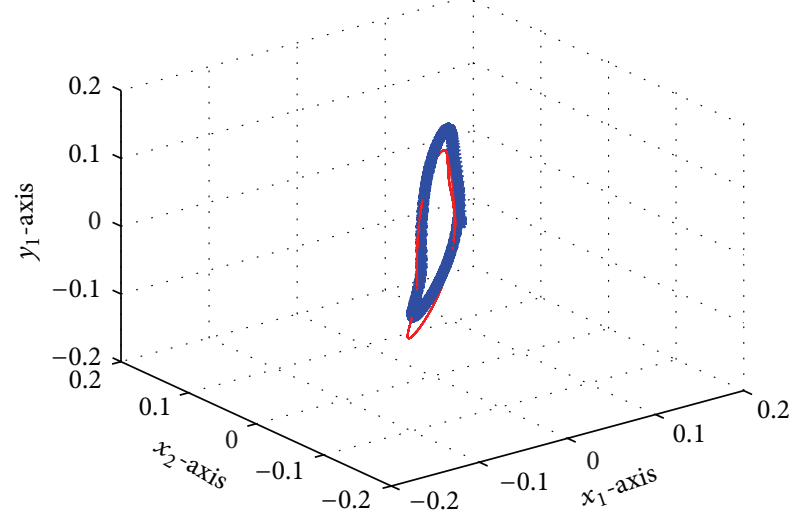

(a)

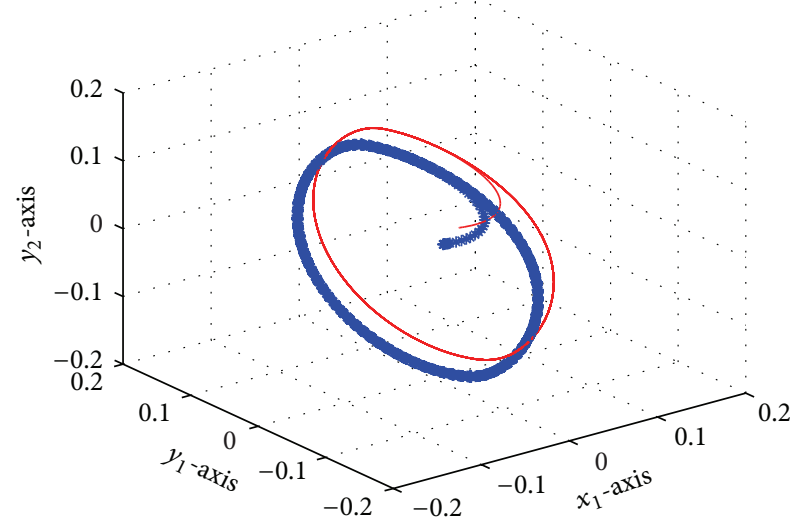

(c)

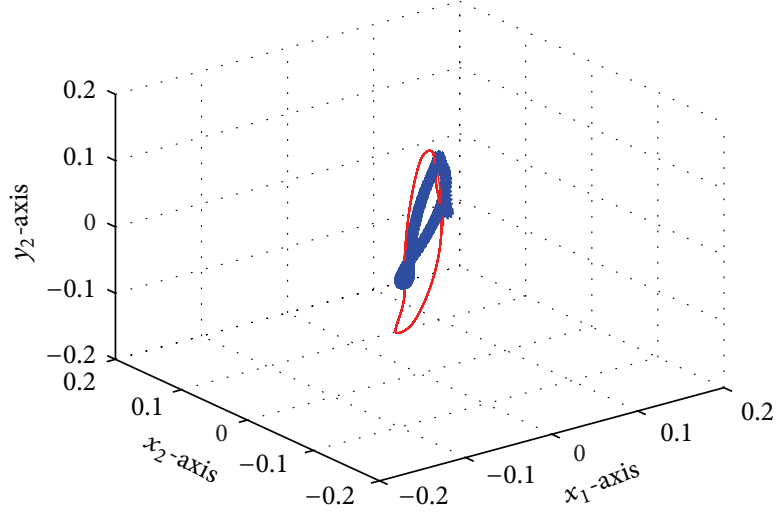

(b)

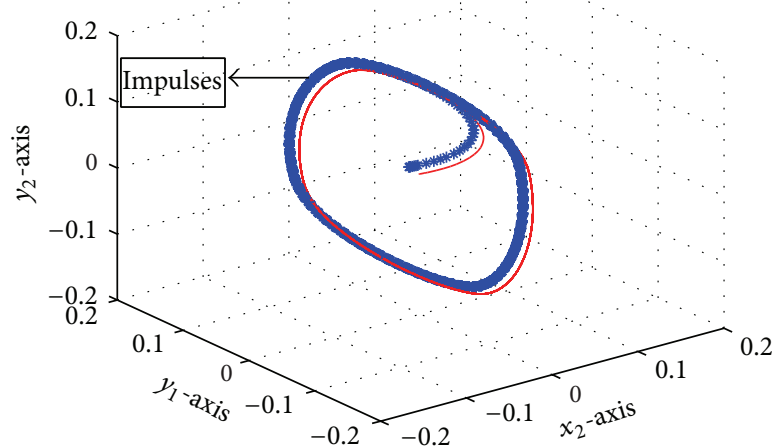

(d)

FIgURE 1: Phase responses of states $x_{1}, x_{2}, y_{1}$, and $y_{2}$.

where $i, j=1,2, f_{j}(x)=g_{i}(x)=(1 / 8)(|x+1|-|x-1|)$, and $t_{1}<t_{2}<\cdots$ is strictly increasing sequences such that $\lim _{k \rightarrow \infty} t_{k}=+\infty$; the coefficients are as follows:

$$
\begin{gathered}
a_{1}(t)=\frac{1}{2}(9+\sin t), \quad a_{2}(t)=4+|\sin t|, \\
\alpha_{1}(t)=\alpha_{2}(t)=\frac{1}{20}(1+\sin t), \\
b_{1}(t)=\frac{1}{2}(9+\cos t), \quad b_{2}(t)=4+|\cos t|, \\
\beta_{1}(t)=\beta_{2}(t)=\frac{1}{20}(1+\cos t), \\
a_{11}(t)=a_{12}(t)=a_{21}(t)=a_{22}(t)=\frac{1}{9}(1+2 \sin t), \\
b_{11}(t)=b_{12}(t)=b_{21}(t)=b_{22}(t)=\frac{1}{9}(1+2 \cos t), \\
d_{11}(t)=d_{12}(t)=d_{21}(t)=d_{22}(t)=\frac{1}{6}(1+\sin t), \\
p_{11}(t)=p_{12}(t)=p_{21}(t)=p_{22}(t)=\frac{1}{6}(1+\cos t), \\
\alpha_{11}(t)=\alpha_{12}(t)=\alpha_{21}(t)=\alpha_{22}(t)=\frac{1}{3} \sin t,
\end{gathered}
$$

$$
\begin{gathered}
\beta_{11}(t)=\beta_{12}(t)=\beta_{21}(t)=\beta_{22}(t)=\frac{1}{3} \cos t, \\
\gamma_{11}(t)=\gamma_{12}(t)=\gamma_{21}(t)=\gamma_{22}(t)=\frac{1}{12}(1+3 \sin t), \\
\eta_{11}(t)=\eta_{12}(t)=\eta_{21}(t)=\eta_{22}(t)=\frac{1}{12}(1+3 \cos t), \\
T_{i j}=H_{i j}=R_{i j}=S_{i j}=1, \\
\omega_{j}=\mu_{i}=1 \quad(i, j=1,2), \\
A_{1}(t)=A_{2}(t)=\frac{1}{2} \cos t, \quad B_{1}(t)=B_{2}(t)=\frac{1}{2} \sin t, \\
\theta_{i k}=1-\frac{1}{3} \sin (2+k), \\
\bar{\theta}_{j k}=1+\frac{2}{3} \cos (3 k), \quad(i, j=1,2) .
\end{gathered}
$$

By calculating, we have

$$
\begin{gathered}
a_{1}^{+}=a_{2}^{+}=b_{1}^{+}=b_{2}^{+}=5, \\
a_{1}^{-}=a_{2}^{-}=b_{1}^{-}=b_{2}^{-}=4, \\
\alpha_{1}^{+}=\alpha_{2}^{+}=\beta_{1}^{+}=\beta_{2}^{+}=\frac{1}{10},
\end{gathered}
$$




$$
\begin{gathered}
a_{i j}^{+}=b_{i j}^{+}=d_{j i}^{+}=p_{j i}^{+}=\alpha_{i j}^{+}=\beta_{i j}^{+}=\gamma_{j i}^{+}=\eta_{j i}^{+} \\
=\frac{1}{3} \quad(i, j=1,2), \\
L_{j}=M_{i}=\frac{1}{4} \quad(i, j=1,2) .
\end{gathered}
$$

We can see that system (32) satisfies the conditions $\left(\mathrm{H}_{1}\right),\left(\mathrm{H}_{2}\right)$, and $\left(\mathrm{H}_{4}\right)$; for $i=1,2, j=1,2$, we have

$$
\begin{aligned}
&-a_{i}^{-}(1\left.-a_{i}^{+} \alpha_{i}^{+}\right)+\left(1+a_{i}^{+} \alpha_{i}^{+}\right) \\
& \times \sum_{j=1}^{2}\left(a_{i j}^{+}+b_{i j}^{+}+\alpha_{i j}^{+}+\beta_{i j}^{+}\right) L_{j}<0, \\
&-b_{j}^{-}\left(1-b_{j}^{+} \beta_{j}^{+}\right)+\left(1+b_{j}^{+} \beta_{j}^{+}\right) \\
& \times \sum_{i=1}^{2}\left(d_{j i}^{+}+p_{j i}^{+}+\gamma_{j i}^{+}+\eta_{j i}^{+}\right) M_{i}<0,
\end{aligned}
$$

which implies that $\left(\mathrm{H}_{3}\right)$ holds. Therefore, from Theorem 3, system (32) is exponentially stable (Figure 1 illustrates our plausible results).

\section{Conclusion}

In this paper, we consider a class of BAM fuzzy cellular neural networks with time-varying delays in leakage terms and impulses. By using differential inequality techniques, we obtain sufficient conditions for the exponential stability of this class of networks. Our results are completely new and complementary to the previously known results. Finally, an example is given to demonstrate the effectiveness and conservativeness of our theoretical results.

\section{Conflict of Interests}

The authors declare that there is no conflict of interests regarding the publication of this paper.

\section{Acknowledgment}

This work is supported by the National Natural Science Foundation of People's Republic of China under Grant 11361072.

\section{References}

[1] B. Kosko, "Bidirectional associative memories," IEEE Transactions on Systems, Man, and Cybernetics, vol. 18, no. 1, pp. 49-60, 1988.

[2] B. Kosko, "Adaptive bi-directional associative memories," Applied Optics, vol. 26, pp. 4947-4960, 1987.

[3] B. Kosko, "A dynamical system approach machine intelligence," in Neural Networks and Fuzzy Systems, pp. 38-108, 1992.

[4] X. Liao, K.-W. Wong, and S. Yang, "Convergence dynamics of hybrid bidirectional associative memory neural networks with distributed delays," Physics Letters A, vol. 316, no. 1-2, pp. 55-64, 2003.
[5] X. F. Liao and J. B. Yu, "Qualitative analysis of bi-directional associative memory with time delay," International Journal of Circuit Theory and Applications, vol. 26, pp. 219-229, 1988.

[6] A. Chen, L. Huang, Z. Liu, and J. Cao, "Periodic bidirectional associative memory neural networks with distributed delays," Journal of Mathematical Analysis and Applications, vol. 317, no. 1, pp. 80-102, 2006.

[7] Z. Zhang, W. Liu, and D. Zhou, "Global asymptotic stability to a generalized Cohen-Grossberg BAM neural networks of neutral type delays," Neural Networks, vol. 25, pp. 94-105, 2012.

[8] R. Samidurai, R. Sakthivel, and S. M. Anthoni, "Global asymptotic stability of BAM neural networks with mixed delays and impulses," Applied Mathematics and Computation, vol. 212, no. 1, pp. 113-119, 2009.

[9] L. Zhang and L. Si, "Existence and exponential stability of almost periodic solution for BAM neural networks with variable coefficients and delays," Applied Mathematics and Computation, vol. 194, no. 1, pp. 215-223, 2007.

[10] Y. Liu and W. Tang, "Existence and exponential stability of periodic solution for BAM neural networks with periodic coefficients and delays," Neurocomputing, vol. 69, pp. 2152-2160, 2006.

[11] L. Hu, H. Liu, and Y. Zhao, "New stability criteria for BAM neural networks with time-varying delays," Neurocomputing, vol. 72, pp. 3245-3252, 2009.

[12] Y. Li and P. Liu, "Existence and stability of positive periodic solution for BAM neural networks with delays," Mathematical and Computer Modelling, vol. 40, no. 7-8, pp. 757-770, 2004.

[13] C. Li, C. Li, X. Liao, and T. Huang, "Impulsive effects on stability of high-order BAM neural networks with time delays," Neurocomputing, vol. 74, pp. 1541-1550, 2011.

[14] P. Balasubramaniam, R. Rakkiyappan, and R. Sathy, "Delay dependent stability results for fuzzy BAM neural networks with Markovian jumping parameters," Expert Systems With Applications, vol. 38, pp. 121-130, 2011.

[15] Y. Li, X. Chen, and L. Zhao, "Stability and existence of periodic solutions to delayed CohenCGrossberg BAM neural networks with impulses on time scales," Neurocomputing, vol. 72, pp. 16211630, 2009.

[16] T. Yang and L.-B. Yang, “The global stability of fuzzy cellular neural network," IEEE Transactions on Circuits and Systems, vol. 43, no. 10, pp. 880-883, 1996.

[17] T. Yang, L. Yang, C. Wu, and L. Chua, "Fuzzy cellular neural networks: theory," in Proceedings of the IEEE International Workshop on Cellular Neural Networks Application, pp. 181-186, 1996.

[18] A. Arunkumar, R. Sakthivel, K. Mathiyalagan, and S. M. Anthoni, "State estimation for switched discrete-time stochastic BAM neural networks with time varying delay," Nonlinear Dynamics, vol. 73, no. 3, pp. 1565-1585, 2013.

[19] P. Vadivel, R. Sakthivel, K. Mathiyalagan, and A. Arunkumar, "Robust state estimation for uncertain fuzzy bidirectional associative memory networks with time-varying delays," Physica Scripta, vol. 88, no. 3, Article ID 035008, 2013.

[20] K. Mathiyalagan, R. Sakthivel, and S. M. Anthoni, "New robust passivity criteria for stochastic fuzzy BAM neural networks with time-varying delays," Communications in Nonlinear Science and Numerical Simulation, vol. 17, no. 3, pp. 1392-1407, 2012.

[21] P. Vadivel, R. Sakthivel, K. Mathiyalagan, and P. Thangaraj, "New passivity criteria for fuzzy BAM neural networks with Markovian jumping parameters and time-varying delays," Reports on Mathematical Physics, vol. 71, no. 1, pp. 69-91, 2013. 
[22] P. Balasubramaniam, G. Nagamani, and R. Rakkiyappan, "Passivity analysis for neural networks of neutral type with Markovian jumping parameters and time delay in the leakage term," Communications in Nonlinear Science and Numerical Simulation, vol. 16, no. 11, pp. 4422-4437, 2011.

[23] X. Li and J. Cao, "Delay-dependent stability of neural networks of neutral type with time delay in the leakage term," Nonlinearity, vol. 23, no. 7, pp. 1709-1726, 2010.

[24] B. Liu, "Global exponential stability for BAM neural networks with time-varying delays in the leakage terms," Nonlinear Analysis: Real World Applications, vol. 14, no. 1, pp. 559-566, 2013.

[25] P. Balasubramaniam, M. Kalpana, and R. Rakkiyappan, "Existence and global asymptotic stability of fuzzy cellular neural networks with time delay in the leakage term and unbounded distributed delays," Circuits, Systems, and Signal Processing, vol. 30, no. 6, pp. 1595-1616, 2011.

[26] X. Li, R. Rakkiyappan, and P. Balasubramaniam, "Existence and global stability analysis of equilibrium of fuzzy cellular neural networks with time delay in the leakage term under impulsive perturbations," Journal of the Franklin Institute, vol. 348, no. 2, pp. 135-155, 2011.

[27] S. Lakshmanan, J. H. Park, H. Y. Jung, and P. Balasubramaniam, "Design of state estimator for neural networks with leakage, discrete and distributed delays," Applied Mathematics and Computation, vol. 218, no. 22, pp. 11297-11310, 2012.

[28] X. Li, X. Fu, P. Balasubramaniam, and R. Rakkiyappan, "Existence, uniqueness and stability analysis of recurrent neural networks with time delay in the leakage term under impulsive perturbations," Nonlinear Analysis: Real World Applications, vol. 11, no. 5, pp. 4092-4108, 2010.

[29] K. Gopalsamy, "Leakage delays in BAM," Journal of Mathematical Analysis and Applications, vol. 325, no. 2, pp. 1117-1132, 2007.

[30] C. Li and T. Huang, "On the stability of nonlinear systems with leakage delay," Journal of the Franklin Institute, vol. 346, no. 4, pp. 366-377, 2009.

[31] P. Balasubramaniam, M. Kalpana, and R. Rakkiyappan, "Global asymptotic stability of BAM fuzzy cellular neural networks with time delay in the leakage term, discrete and unbounded distributed delays," Mathematical and Computer Modelling, vol. 53, no. 5-6, pp. 839-853, 2011.

[32] Y. K. Li, L. Yang, and L. J. Sun, "Existence and exponential stability of an equilibrium point for fuzzy BAM neural networks with time-varying delays in leakage terms on time scales," Advances in Difference Equations, vol. 2013, article 218, 2013.

[33] V. Lakshmikantham, D. D. Bainov, and D. Simeonov, Theory of Impilsive Differential Equations, World Scientific, Singaore, 1989.

[34] Y. Li, "Global exponential stability of BAM neural networks with delays and impulses," Chaos, Solitons and Fractals, vol. 24, no. 1, pp. 279-285, 2005.

[35] X. Liu and G. Ballinger, "Existence and continuability of solutions for differential equations with delays and state-dependent impulses," Nonlinear Analysis: Theory, Methods \& Applications, vol. 51, no. 4, pp. 633-647, 2002.

[36] T. Yang, L. Yang, C. Wu, and L. Chua, "Fuzzy cellular neural networks: applications," in Proceedings of the IEEE International Workshop on Cellular Neural Networks Application, pp. 225-230, 1996. 


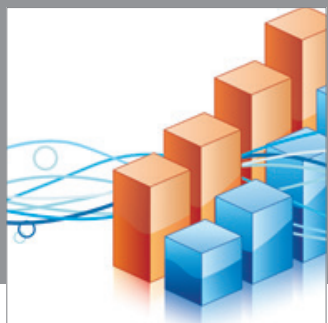

Advances in

Operations Research

mansans

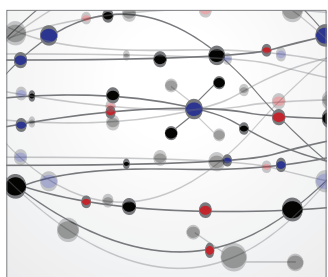

The Scientific World Journal
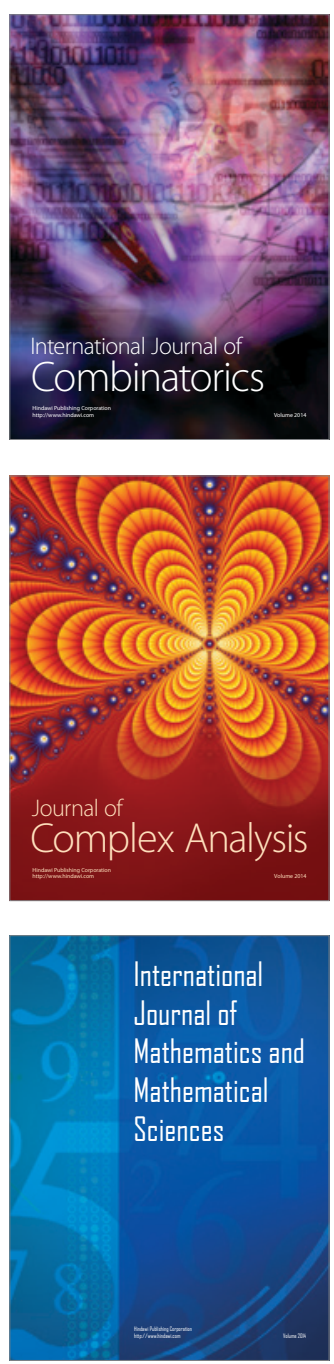
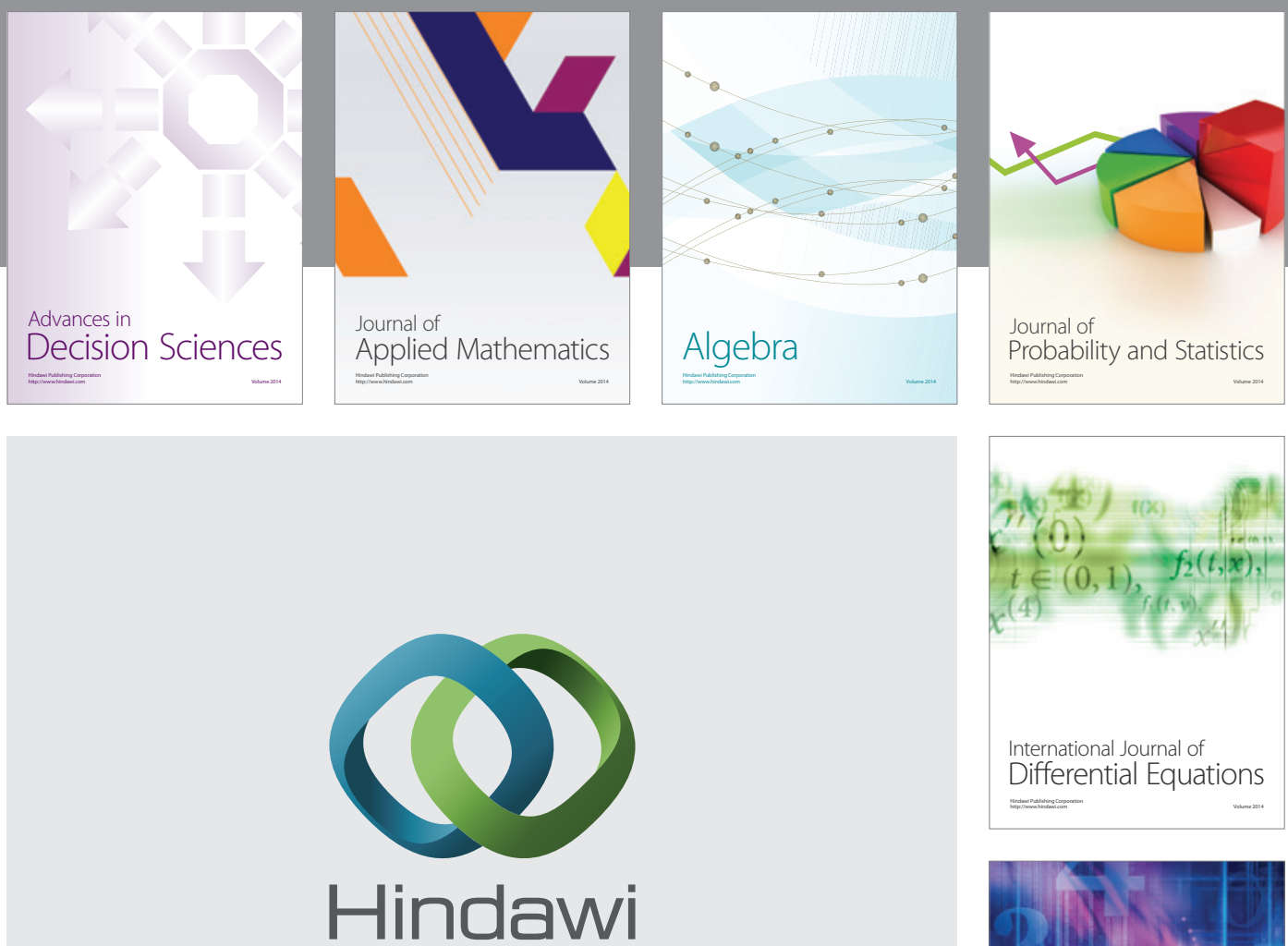

Submit your manuscripts at http://www.hindawi.com
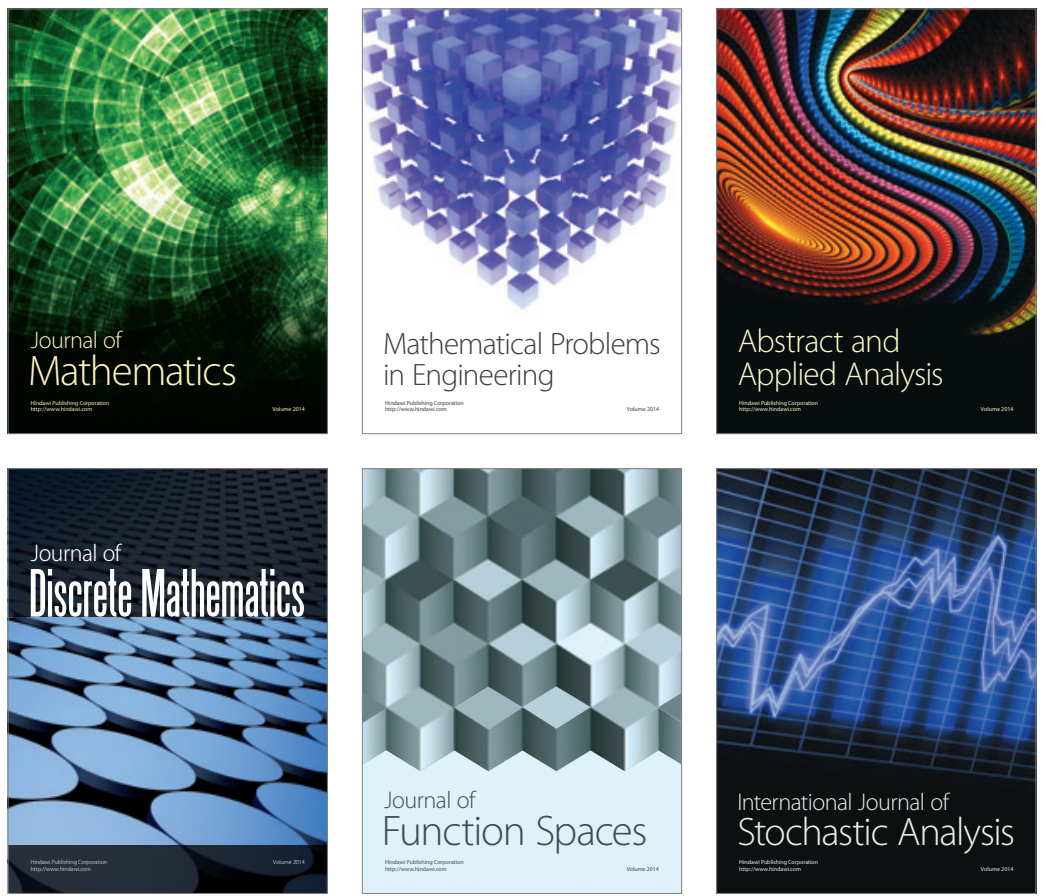

Journal of

Function Spaces

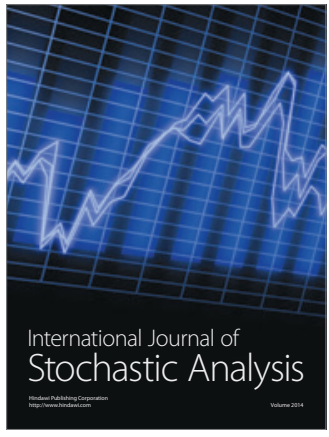

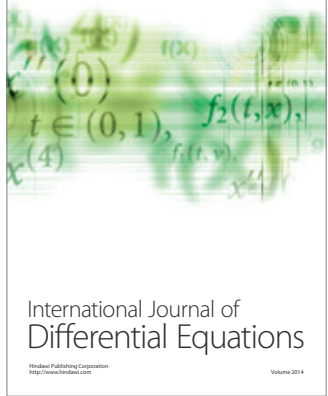
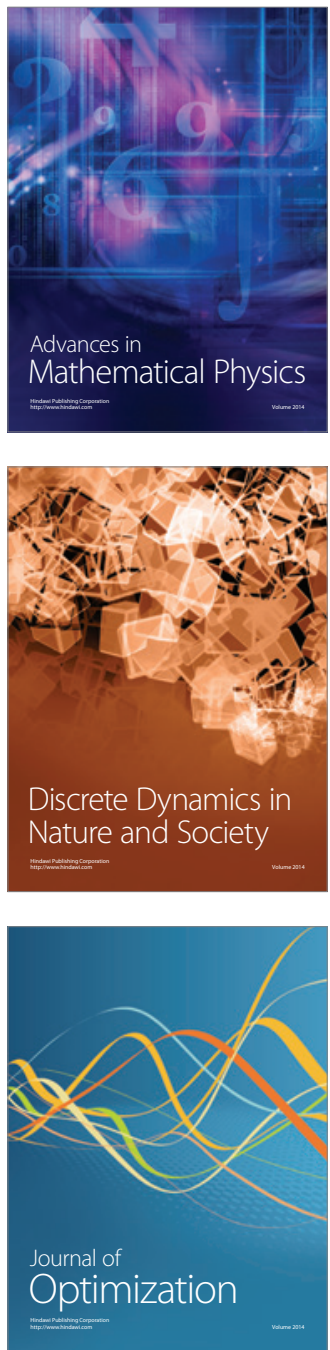